\title{
Developing a Low-Cost, Ultraportable, Modular Device Platform to Improve Access to Safe Surgery
}

\section{Citation}

Teodorescu, Debbie. 2016. Developing a Low-Cost, Ultraportable, Modular Device Platform to Improve Access to Safe Surgery. Doctoral dissertation, Harvard Medical School.

\section{Permanent link}

http://nrs.harvard.edu/urn-3:HUL.InstRepos:40620229

\section{Terms of Use}

This article was downloaded from Harvard University's DASH repository, and is made available under the terms and conditions applicable to Other Posted Material, as set forth at http:// nrs.harvard.edu/urn-3:HUL.InstRepos:dash.current.terms-of-use\#LAA

\section{Share Your Story}

The Harvard community has made this article openly available.

Please share how this access benefits you. Submit a story. 


\section{Scholarly Report submitted in partial fulfillment of the MD Degree at Harvard Medical School}

Date: 1 March 2016

Student Name: Debbie Lin Teodorescu, AM, MEng

Scholarly Report Title: Developing a Low-Cost, Ultraportable, Modular Device Platform to Improve Access to Safe Surgery

Mentor Name and Affiliation: Christiana A. Iyasere, MD, MBA, Dept of Medicine, Massachusetts General Hospital

\section{Collaborators, with Affiliations:}

Dennis Nagle, D-Lab, Massachusetts Institute of Technology

José Gómez-Márquez, Little Devices Lab, Massachusetts Institute of Technology

Anna Young, Little Devices Lab, Massachusetts Institute of Technology

Madeline Hickman, D-Lab, Massachusetts Institute of Technology

David R. King; Division of Trauma, Acute Care Surgery and Surgical Critical Care;

Massachusetts General Hospital 


\section{Abstract}

TITLE: Developing a Low-Cost, Ultraportable, Modular Device Platform to Improve Access to Safe Surgery

Debbie L. Teodorescu, Dennis Nagle, José Gómez-Márquez, Anna Young, Christiana A. Iyasere, Madeline Hickman, David R. King

Purpose: A third of the global disease burden requires surgical therapy, yet in disaster-affected areas and lower-middle income countries, 5 billion people have little or no access to safe surgical care. We develop, evaluate, and aim to deploy a new technology to help increase access to safe surgery.

Methods: We conducted a needs assessment, then used iterative prototyping to incorporate stakeholder feedback and testing of all components. Proof-of-concept testing of the prototype entailed setup over a mannequin and using a particle counter to evaluate ability to provide a contaminant-free sterile field.

Results: Our prototype shrinks the scope of the sterility challenge from the room to the critical space immediately over the incision. Users seal the modular system of sterile clear containers over the patient and operate via ports. An integrated airflow system controls enclosure conditions. Everything folds for rapid deployment. Testing demonstrates superior environmental control compared to standard operating rooms, including setup time, time to surgical site sterility, resistance to active contamination, and air changes per hour.

Conclusions: Our results indicate that it is possible to provide state-of-the-art levels of sterility during surgeries even in austere settings, by using a low-cost, ultraportable, modular system codeveloped with key stakeholders. 


\section{Student Role}

The author is the project leader. The role comprises personally and collaboratively accomplishing the following key tasks:

1) Prototype design

2) Prototype fabrication

3) System modeling (in silico and analytical)

4) Experimental protocol design and modification for in vitro, in vivo, and ex vivo field testing

5) Prototype optimization

6) Producing and seeking input on the major drafts of posters, paper manuscripts, grants, and other key scholarly output.

7) Working with other members of the development team, key collaborators, and stakeholders to advance project goals.

8) Recruiting and working with new members of the development team and collaborators to meet challenges in the SurgiBox development cycle. 
From UNESCO-EPFL Tech4Dev Conference 2016

http://cooperation.epfl.ch/files/content/sites/cooperation/files/1171-Teodorescu-SE07-

MED Full\%20Paper.pdf

\title{
Developing a Low-Cost, Ultraportable, Modular Device Platform to Improve Access to Safe Surgery
}

Debbie L. Teodorescu ${ }^{1,2}$, Dennis Nagle ${ }^{1}$, José Gómez-Márquez ${ }^{3}$, Anna Young ${ }^{3}$, Christiana A. Iyasere $^{2,4,5}$, Madeline Hickman ${ }^{1}$, David R. King ${ }^{2,6,7, *}$

${ }^{1}$ MIT D-Lab, Cambridge, United States of America

${ }^{2}$ Harvard Medical School, Boston, United States of America

${ }^{3}$ MIT Little Devices Lab, Cambridge, United States of America

${ }^{4}$ Massachusetts General Hospital Department of Medicine, Boston, United States of America

${ }^{5}$ Massachusetts General Hospital Innovation Task Force, Boston, United States of America

${ }^{6}$ Massachusetts General Hospital Division of Trauma, Acute Care Surgery and Surgical Critical Care; Boston, United States of America

${ }^{7}$ Massachusetts General Hospital Knight Surgical Research Laboratory, Boston, United States of America

\begin{abstract}
:
Surgery saves lives in traumas, obstetric emergencies, infections, oncology, and more. Indeed, $30 \%$ of the global disease burden requires surgical therapy, yet in lower-middle income countries, 5 billion people have little or no access to safe surgical care. Costly infections from unsterile surgeries threaten developing infrastructures. Safe surgery is thus a global health priority. SurgiBox, a joint project of MIT D-Lab and Massachusetts General Hospital, aims to develop, evaluate, and ultimately deploy a new technology to help increase access to safe surgery. SurgiBox shrinks the scope of the sterility challenge from the room to the critical space immediately over the incision. Users seal the modular system of sterile clear containers over the patient and operate via ports. An integrated airflow system controls enclosure conditions. Everything folds for rapid deployment. This project requires close dialogue among stakeholders with iterative, rapid prototyping changes. Benchtop and simulation testing to date demonstrate superior environmental control compared to standard operating rooms, notably including setup time, time to surgical site sterility, resistance to active contamination, and air changes per hour. Ongoing efforts include testing in stress use scenarios to replicate field conditions, field testing, in vivo testing, minimizing device cost, and mapping out a sustainable deployment and scale-up strategy.
\end{abstract}

Keywords: Global surgery, safe surgery, SSIs, LMIC, technological innovation

\section{Full Paper}

\section{Introduction}

Over $30 \%$ of the global disease burden requires surgical therapy, which could prevent over 18 million deaths and save USD \$200 billion annually. The conditions amenable to surgical therapy range broadly, from traumatic to obstetrical to infectious to oncological and beyond. Yet in lowmiddle income countries (LMICs), an estimated 2 billion people have effectively no access to 
surgical care, and another 2-3 billion have access only to surgeries performed in unsterile settings such as general-use buildings or even outdoors ("Global Surgery 2030", 2015; Disease Control Priorities Project, 2008). In addition to this chronic deficiency in surgical access, field surgical zones in disaster-affected areas are often exposed to frank particulate and insect contamination.

In LMICs, surgical patients develop disproportionate rates of surgical site infections (SSIs), particularly the deep infections characteristic of intraoperative contamination. Meta-analyses (Allegranzi et al 2011) have found that 0.4-30.9 per 100 surgical patients in LMICs develop SSIs. In particular, even in clean and clean-contaminated wounds, which had not previously been contaminated by traumatic skin breaks, uncontrolled gut flora spillage, etc, the median cumulative incidences were still respectively $7.6 \%$ (range $1.3-79.0 \%$ ) and $13.7 \%(1.5-81.0 \%$ ), all several times higher than in higher-income countries (Ortega et al 2012). Most alarmingly, these figures represent early postoperative infections of deep visceral spaces and organs, not superficial tissues, a finding underscored by Nejad et al's (2011) meta-analysis that showed $6.8-46.5 \%$ incidence of deep infections in postoperative patients, and 10.4-20.5\% of infections in organ spaces. Bjorklund et al's 2005 analysis showed a particularly unfortunate interaction between immunosuppression all too common in the developing world due to poor nutrition, untreated illness, and HIV - and unsterile surgical conditions in producing very high rates of severe infection following c-sections. These infections translate into longer stays at already-overcrowded hospitals: 8 additional days on average in Tanzanian and Ethiopian studies, 10 days in a Burkina Faso study comparing surgical patients with and without SSIs (Eriksen et al 2003, Taye 2005, and Sanou et al 2009). In nascent healthcare systems with limited infrastructures, SSIs that effectively double or triple patient stay lengths fetter institutions' ability to cope and reduce the volume of new patients that could be accommodated. Taye (2005) noted that SSIs were associated with 2.8 -fold increased mortality $(10.8 \%$ vs $3.9 \%)$.

Numerous factors impact surgical site infection rates. These have been most authoritatively summarized by the Lancet Commission on Global Surgery (2015) and range from preoperative antibiotic administration to drape selection to handwashing and beyond. A particularly pernicious and challenging one to address has been that of the contaminated environment. Whyte et al (1982) and Edmiston et al (2005) have described the general link between airborne contamination and SSIs, with an estimated $30 \%-98 \%$ of wound bacteria attributable to airborne contaminants, depending on the ventilation system in an operating room. In higher-income countries, invasive procedures are typically performed by scrub-attired personnel striving to reduce contamination in operating rooms with meticulously-filtered air. In LMICs, such facilities and infrastructure are frequently unavailable. Procedures instead often occur wherever dedicated space could be found, whether general-use rooms, outdoors, or other suboptimal settings. Pathogen-carrying insects, dust particles, provider skin squames, and numerous other dangers frequently breach the sterile field. Even in state-of-the-art operating rooms, relatively modest breaches due to events such as door openings have been associated with increased SSI rates. Indeed, decreasing the number of times doors were opened decreased SSI rates by $36 \%$ in one study and 51\% in another (Van der Slegt et al 2013 and Crolla et al 2012). The absence of any door at all, or of effective surgical suite ventilation, in the LMIC operating space is therefore quite a concern. 
The need to provide safe surgical care outside of traditional surgical facilities is certainly not a new problem. However, solutions to the challenge have typically started from the assumption that the core problem is to provide a sterile operating room outside of a standard facility. This mindset informs solutions such as surgical tents, operating rooms mounted on trailers or trucks, semiportable laminar airflow systems, and most other solutions to date. These devices unfortunately tend to share several significant limitations in practice. They are challenging to transport to remote or disaster-affected areas, requiring both time and logistical capability. Once at the desired site, they require significant setup time. For example, surgical tents can take a full team of technicians working around the clock for 72 hours to fully set up. Several of these systems have at least one external dependency, particularly availability of electricity or requirement of flat terrain. They require significant resources not only for sunk cost, but also for marginal cost of each procedure. Personnel, a particularly scarce resource, are also required to set up and maintain these complex systems. These systems are not always robust to the high levels of external contamination, with sand particulate ingress into the tents a particularly notorious phenomenon in the field (e.g. as described by Stevenson and Cather 2008). Finally, any contamination in these systems, including that generated by providers through squame shedding, can still contaminate the surgical site.

The SurgiBox platform moves away from the assumption that the surgical space of interest is the operating room. Fundamentally, the space that matters is the incision and immediate surgical field over the patient. This recognition literally shrinks the challenge down from over thousands of cubic feet of space to well under 10 cubic feet to be kept sterile. The nature of protection actually changes, as contamination can come from patients themselves, providers, and the external environment. Creating a physical barrier from contamination completely from the latter two, and significantly from the patients themselves, theoretically permits more robust protection than would the classic, costly combination of sterile room, sterile scrub suit, and sterile drapes. A system that can effectively maintain a sterile field in this limited space, as presented here, provides a low-cost, ultraportable platform for regulating intraoperative conditions at the surgical site, making safe surgery more accessible.

This paper is organized as follows. Section II describes SurgiBox's iterative prototyping and evaluation methods. Section III presents the design and our results to date. Section IV discusses ongoing as well as future efforts in device development and deployment. Section V discusses SurgiBox's broader implications.

\section{Methods}

\subsection{Patient- and stakeholder-centered development}

The objective of SurgiBox overall is to provide a low-cost, ultraportable means by which to maintain sterile conditions during invasive procedures, even when performed in contaminated settings. However, as with any medical device, particularly one intended for developing settings, other complex requirements are critical to stakeholder acceptance (Caldwell et al 2011). To mitigate what we perceived as a key risk in this project, that of rejection of the device by end-users despite technical success, we conducted extensive preliminary as well as ongoing stakeholder interviews with physicians who work in the developing world, surgical researchers, biomedical and device engineers, global health and development researchers as well as other workers, members of the medical device industry, and innovation strategists. These discussions generated the objectives and specifications as shown in Table 1. We used existing data from anthropometric 
tables (with the aim to accommodate the $5^{\text {th }}$ through $95^{\text {th }}$ percentiles of providers and of patients), surgical ergonomics research, and operating room design guidelines to populate design specifications for the prototypes.

\section{Table 1. Stakeholder-Generated Device Specifications}

\begin{tabular}{|l|}
\hline $\begin{array}{l}\text { Ultraportable - can be deployed via modalities with minimum space, such as manual baggage or even } \\
\text { unmanned aerial vehicles (both weight and packaged bulk matter) }\end{array}$ \\
\hline Rapid assembly, disassembly, and reassembly; would not significantly change workflow \\
\hline Accommodates wide range of patient body habitus \\
\hline $\begin{array}{l}\text { Compatible with open surgical procedures on any portion of the patient torso (for the first version; } \\
\text { head/extremities and minimally-invasive modalities are important as well for future versions) }\end{array}$ \\
\hline $\begin{array}{l}\text { Permits up to two users working in the surgical field directly, with the option for more via integrated } \\
\text { modules; but only requires single user to function }\end{array}$ \\
\hline Ergonomic for wide range of surgical and surgical assistant activities, with varying user sizes \\
\hline Ports for arms and material transfers that are both ergonomic and able to maintain sterile field \\
\hline Ports for arms must permit intraprocedural glove exchange in case of damage \\
\hline Excellent visual clarity of the field to varying lighting conditions \\
\hline Maintain sterile field in diverse stress conditions \\
\hline Passive barrier to body fluid splash injury to user \\
\hline Adequate humidity control to prevent condensation \\
\hline $\begin{array}{l}\text { Able to meet or exceed state-of-the-art operating room regulatory guidelines with regards to active air } \\
\text { quality control }\end{array}$ \\
\hline Reusable components are durable to stress, shocks, temperature extremes, and dust. \\
\hline Low marginal use cost for each surgical case (well under USD \$20 per case). \\
\hline Ready to use as is: no dependency on external resources such as electricity \\
\hline Modular in nature
\end{tabular}

Figure 1 sketches out our group's iterative process of design, prototyping, testing, and design optimization. By optimizing key components of the system ("wall/frame", "ports", and "environmental control") in parallel, we were able to minimize resource waste, more efficiently engage the various members of a diverse development team, and more rapidly elicit then respond to feedback as well as small-scale testing.

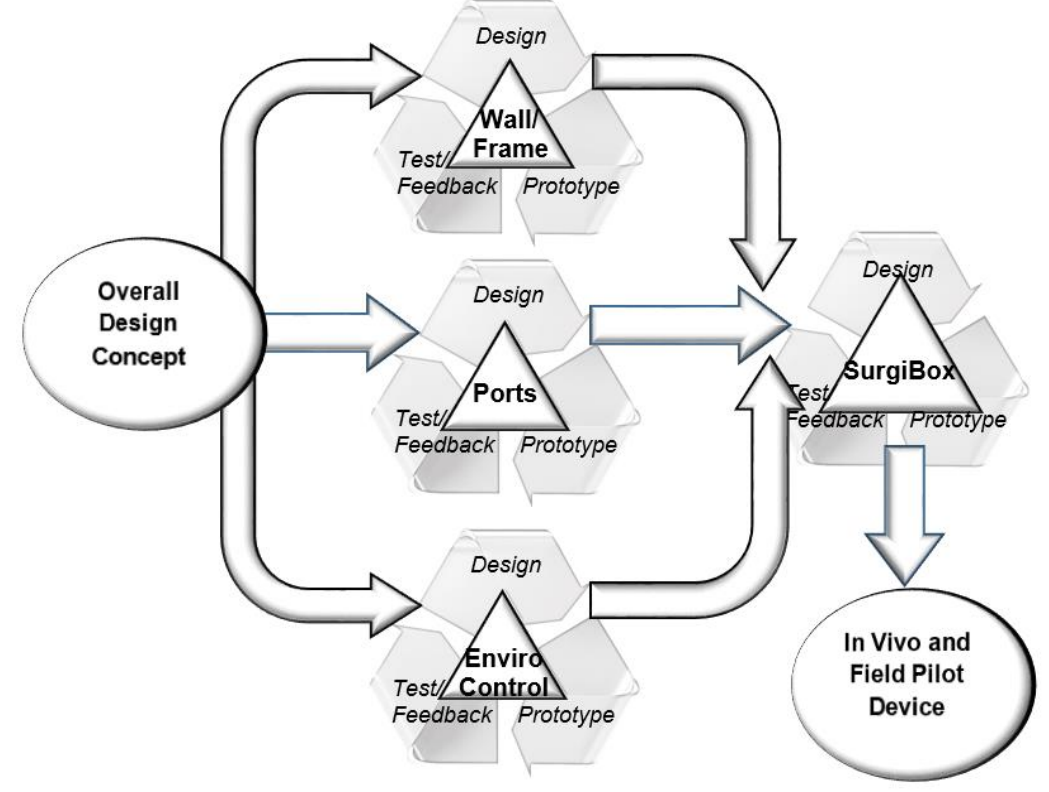

Figure 1. Schematic of SurgiBox's co-development process.

\subsection{Proof of concept testing}


Though ongoing evaluation is central to the co-development process for all aspects of SurgiBox, the key technical question is: can the system actually provide a level of sterility consistently equivalent to or exceeding that available in state-of-the-art operating rooms?

The location for testing was selected to be a mixed-use machine shop at the MIT D-Lab. To standardize testing, we set up three different rigs to replicate three types of modules: one with completely intact sides (corresponding to fully-sealed systems), one with a 6-inch open slit on each side of the patient flank-contacting sides (corresponding to systems with easy-access material ports for taking supplies and specimens on or off the sterile field), and one with both the material ports and a 20 -inch horizontal slit on each side of the patient flank-contacting sides (corresponding to systems with open arm ports as well as easy-access material ports). The rigs were set up by mounting the disposable component to the rigid frame, and then tucking the disposable component around a plastic mannequin, allowing gaps. Airflow was provided through a dedicated air inlet above the head of the mannequin. Stress conditions were simulated by producing talcum powder puffs around all of the sides of the rig with intact sides. All particle counts were obtained from a P-Trak model 8525, with probe positioned either at the mannequin xiphoid or alternating flanks.

\section{Results}

\subsection{Device Design}

SurgiBox is an ultraportable, modular system to provide sterile intra-operative environments over surgical sites. This idea's embodiment went through extensive evolution. Briefly, we initially designed a box-like system comprising hinged clear panels of polycarbonate that could be collapsed into a flat package. This system could be reprocessed and reused. Based on stakeholder feedback regarding sterilization capabilities, bulk, ergonomics, and modularity potential, we changed the design to combine a disposable, patient-contacting component walls with a reusable component comprising the frame and environmental controls. We had also designed open arm ports to permit surgeons and assistants to insert their gowned arms, but subsequently changed to glove-sealed arm ports based on feedback regarding perceived cleanliness of reentry, field specialists' interest in reducing personnel garb costs, and testing that showed significantly higher costs of environmental control. By contrast, whereas we had originally planned for material ports to be hermetic airlock-inspired systems, feedback on impact to workflow and reassuring testing prompted redesign to quick-opening ports with a single layer of sealing. After trialing several different systems including recirculation, dessication, and temperature changing systems, the main modality of environment control was elected to be HEPA-filtered laminar airflow, which additionally controls humidity levels. Whereas American operating room design guidelines require at least 15 air changes per hour with 3 of external air for standard operating rooms (American Institute of Architects 2006), we supplied the capability for up to 90 air changes per hour.
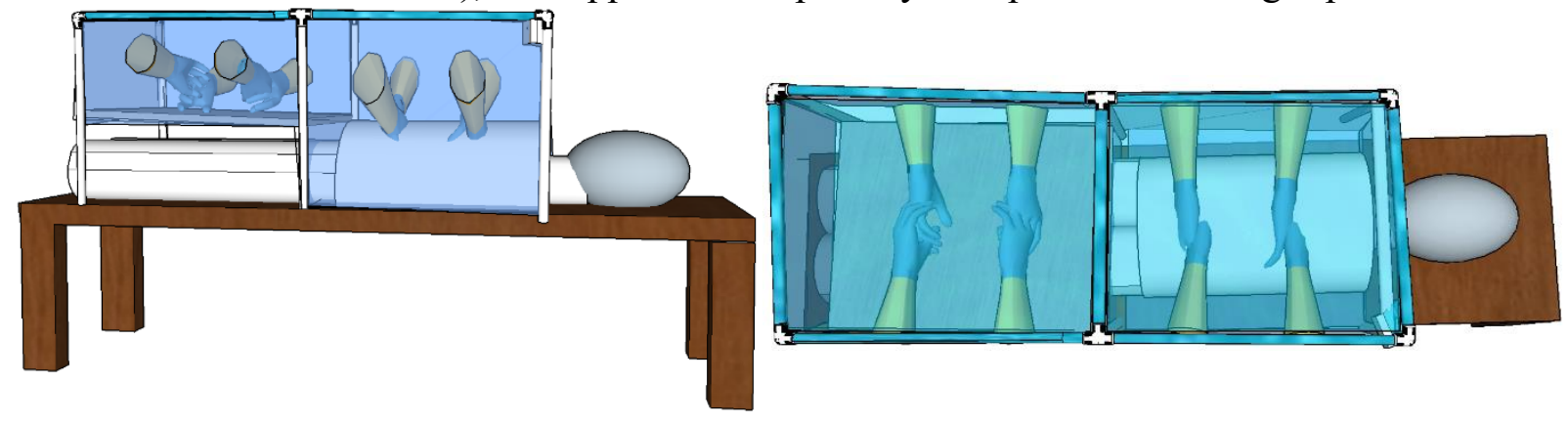

Figure 2a. Side view of SurgiBox with the incision protection and Figure 2b. Top view of SurgiBox which is the surgeons' and assistants' 
Figure 2 shows the computer-aided design images of early versions of SurgiBox, which comprised two modules, one for the incision site and one for a dedicated instrument tray. These can be connected to form a continuous sterile field but can also be used independently. Both include a flexible clear film covering the surgical field, mounted to a rigid, collapsible, resizable frame. The sterile field is accessed via two pairs of arm ports on each module, as well as two material transfer ports. Tension of the superior wall permits clear visual field from above. The left module, shown overlying the patient's legs in Figure 2, holds an instrument tray. Many other modules are possible including to replace back tables, permit laparoscopy, etc. Environmental controls are integrated into the rigid frame and together comprise the reusable portion of the device; the clear portion is changed between patients.

\subsection{Particle Testing}

Design freezes based on acceptable ergonomics and visual field clarity were determined following multiple rounds of testing and discussions among surgical and engineering members of the team. Benchtop testing with the particle counter demonstrated an average of $22.8+/-2.8 \%$ reduction in particle count between the outside and inside of the SurgiBox five minutes after the system was set up in a nonsterile manner, using purely passive barrier (i.e. with the environmental regulation system off). The internal particle count did not significantly change following introduction of sufficient external talcum particles to cause a $28.3 \%$ concentration increase at the SurgiBox walls. Engaging of the airflow system, even when the SurgiBox itself was set up in nonsterile fashion with initially contaminated enclosed air, consistently produced 0 particle counts in less than 95 seconds as shown in Figure 3, measured at both the xiphoid and flanks on the test mannequin, even when ports were simply cut out without valves or barriers. This corresponds to the purging period after each patient setup. Low airflow was required to maintain the particle counts at 0 . Together, these results prove the concept of achieving state-of-the-art level sterility in contaminated environments.

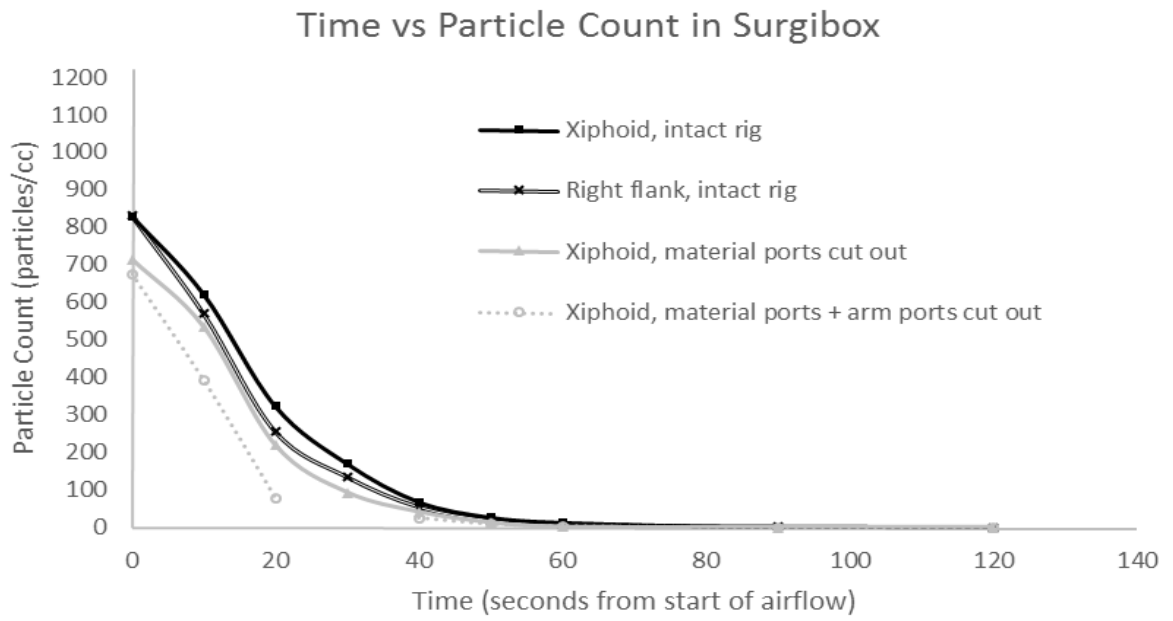

Figure 3. Results from testing on dedicated rigs on a standardized mannequin, using a particle counter located at either the xiphoid or the flanks, areas of particular interest for abdominal, pelvic, and thoracic procedures. The rigs comprised three increasing levels of communication with the external environment ("intact rig", "material ports cut out", and "material ports + arm ports cut out") were either mounted over the patient directly with only gaps at the bottom from tucking or had a vertical slit cut in each long side for material port or had both a vertical slit and a long horizontal slit for arms port. Airflow purged all of these setups of particles. 


\section{Discussion}

\subsection{Ongoing and Future Research}

Our efforts to further optimize SurgiBox with our existing iterative prototyping workflow are ongoing. We are particularly interested in further reducing the cost of the system, which currently runs up to USD \$1,150 with off-the-shelf components for maintaining environmental control. The cost-determining portions are fully reusable between patients for an estimated 10,000 cases depending on airflow requirement, based on the life expectancies of the battery and the filter cartridge, both of which are replaceable to permit continued use of the system. Ongoing research is combining two strategies to reduce these costs: reducing maintenance airflow requirement by improving the system's integrity and reducing air leakage; and designing a system to run on either mechanical mechanisms or standard batteries instead of proprietary ones. Such a system would be expected to cost under USD $\$ 40$ total. The patient-contacting portions currently cost approximately USD \$5 each when produced in small quantities, though current efforts to convert the material to lower-cost, environmentally-friendlier low-density polyethylene should further reduce the cost. These components are ideally disposable, but given what we understand thus far to be limited and mitigatable cross-contaminating potential, reuse following reprocessing may be acceptable.

In addition, the current experimental setup has two main limitations. First, although particle testing is a well-validated way to obtain dynamic measurements of the system's barrier function, we also plan to correlate with settle plate testing to assess colony forming unit counts. The second limitation is that the tests are conducted in static conditions. Ongoing work is using standardized rigs to simulate use conditions.

While we do strive to simulate field conditions in the lab and to assess ease of use of the prototypes by stakeholders with experience in LMIC settings, we are planning to work with LMIC community partners abroad to supply SurgiBox kits to the field. By performing ex vivo setups in truly field conditions, we can collect further data and feedback to identify any additional issues requiring optimization as well as impacts on workflow. By supplying SurgiBox kits with a variety of frame and port components, we hope to encourage community partners to tinker with the prototypes to suit their needs.

While we have striven at every step to make SurgiBox as user-friendly as possible, we recognize there will likely be resistance if it were presented on the basis of in vitro sterility data only. After all, there are many determinants of safe surgery. Therefore, even though SurgiBox would qualify as a United States Food and Drug Administration Class I device that does not require efficacy studies, we still plan to voluntarily conduct trial surgeries on animals in simulated field conditions to assess impact on clinical outcomes such as wound contamination rates and SSI rates.

\subsection{Application in Practice}

At the same time, we are actively evaluating best practices for deploying SurgiBox. Our major considerations fall into three categories: market segmentation, production, and distribution.

\subsubsection{Market Segmentation}

One unexpected finding from stakeholder interviewers was the extent to which the device platform

offers potential value to settings through the whole spectrum of resource availability. Practitioners 
in austere LMIC settings recognize its ability to provide a sterile environment and potentially reduce reliance on facilities as well as scrubs or drapes. In higher-resource settings, it may reduce healthcare costs by permitting procedures to be performed in less-expensive procedure rooms, or maybe reduce SSIs if used in tandem with existing facilities. In tertiary care centers, it can conceivably offer improved outcomes in lengthy, complex procedures by providing more intensive control of the intraoperative environment.

Ultimately, these discussions shaped SurgiBox's design. Because we are committed to the austere setting to maximize direct patient benefit, our design creates a cost-minimizing, minimum viable product. Yet recognizing the potential value of other future market segments, we also developed the system to be highly modular, to permit subsequent adaption to other settings. In global development, it is well-understood that it can be advantageous to simultaneously develop different tiers of product, with higher-profit sales in higher-income market segments helping to finance products for LMIC sectors. In SurgiBox's case, we moved away from this strategy in favor of successive expansion because of the considerable resources needed for successful deployment of a surgical device in any setting, particularly one that challenges existing paradigms and workflows.

Within the broad market segment of austere surgery, we expect the early adopters to be surgeons from higher-income countries working in LMICs. The driving reason is that within this business development model, we would be proposing to introduce to LMICs a technology that does not yet have an established equivalent in higher income countries. Our justification is that this addresses a distinctive problem - unsafe intraoperative environment - in these settings. Although data support the value proposition, our stakeholder interviews revealed that perception of the problem differs among users. The most concern came from practitioners splitting their practice between higher-income and LMICs, that they are unable to provide the standard of hygiene to which they are accustomed to their patients in the latter. The problem, to them, is a graphic one with clear implications for providing "good" care. In addition, these physicians note another benefit to SurgiBox: protecting the provider from aerosols and splashes. And indeed, during the Ebola outbreak, it was higher-income countries' physicians and organizations that promulgated and implemented standards for appropriate drapes and protective equipment for operating on possiblyinfected patients.

Accordingly, the first purchasers would be entities supplying providers of surgical aid in disaster relief and chronically low-resource settings. Market analysis with pricing remains ongoing. In the long term, we plan to engage with LMIC health ministries to deploy the device to locally-based surgeons to encourage sustainable development.

\subsubsection{Production}

To support advanced development of SurgiBox and pilot deployment, our collaboration is able to leverage our position at the intersection of academia and nongovernmental organization to seek both competitive grant funding and mutually-beneficial partnerships with other nongovernmental or governmental organizations. As a social venture, we further benefit from the robust small business and social entrepreneurship resource infrastructure available in the United States in general and in the Boston area in particular, including funding initiatives. 
SurgiBox's design and prototyping process have emphasized minimizing not only the cost of raw materials but also of manufacturing and packaging. By finding alternatives to complex, highvariance, high-cost components, we expect that the device should be able to be manufactured to consistently high quality by a contract or co-development entity's manufacturer able to comply with United States Federal Drug Administration General Controls and similar regulations. Indeed, it is conceivable that we can eventually engage with regional or local manufacturing entities during scale-up stage, recognizing the importance of maintaining quality control compatible with a medical device. With the "cost of goods sold" for benchtop prototypes stabilizing, we are now conducting this analysis in the real world setting.

\subsubsection{Distribution}

SurgiBox itself will be distributed as ultraportable kits suitable for hand luggage, backpacks, drones, and other limited spaces. These kits will contain the reusable component, one or more of the patient-contacting components all individually wrapped, and batteries: all the items needed for full use of the system. Users can therefore continue to utilize their preferred skin disinfectant, lighting source, instrument trays, gloves on top of the universal-sized thinner gloves in place, and other things to best preserve workflow.

At this stage, we are still working on developing strategic partnerships critical to pilot deployment and eventually distribution success in the future. Supply and demand bottleneck analyses of the expected uptake challenges along the value chain are ongoing, as highlighted above. In the market segment we are targeting, procurement is primarily by each mission-sponsoring entity - most commonly militaries, surgical relief organizations, hospitals, and device companies - or by individual providers. Upstream, many of the former have relationships with procurement superstructures such as the World Health Organization, which in 2015 reported allocating the plurality (\$333 million) of its procurement budget to strategic category products, which cover most key surgical devices, tools, and kits. Certainly, engaging with all of these diverse stakeholders is critical to success.

\section{Conclusion}

Taken together, the growing interest in surgery as an inalienable part of global health, as well as the ethical as well as practical need to provide this surgical care in a safe manner, provides a rich opportunity for innovative solutions to the complex challenges entailed.

In this paper, we described one such innovation in the form of a co-designed, ultraportable sterile field platform. While developing overall surgical infrastructure and facilities are critical ultimate objectives, we refuted the assumption that protecting patients from airborne intraoperative contamination relies on achievement of those objectives. By shifting the site of regulation from the operating theater to the incision itself, we introduced a novel paradigm more amenable to flexible, cost-effective solutions.

To reduce this paradigm to practice, we closely engaged user-stakeholders by starting with a systematic needs analysis, then using feedback to drive the evolution and refinement of SurgiBox. We presented the device design and results from benchtop testing that showed how SurgiBox can rapidly create a particle-free environment. Ultimately, deploying SurgiBox to LMICs and beyond 
requires continued close stakeholder engagement in the form of robust relationships along the production and supply chains.

\section{Acknowledgements}

We thank D-Lab's Amy Smith and Victor Grau Serrat for consistent support over the years. Stephen Odom and Dana Stearns have provided key design input and implementation insights. We are further grateful to Marissa Cardwell, Jim Doughty, Fabiola Hernandez, and Kerry McCoy of MIT's Environmental Health Services for technical assistance and device design feedback. Kristian Olson offered invaluable advice on implementation and deployment strategy. Jack Whipple has provided machine shop assistance. This work was supported by the Harvard Medical School Scholars in Medicine Office.

\section{References}

\section{Book chapter}

American Society of Heating, Refrigeration and Air-Conditioning Engineers (2011). Health Care Facilities (I-P). In ASHRAE 2011 Handbook - HVAC Application. Atlanta: ASHRAE.

\section{Journal article}

Allegranzi, B.,Bagheri Nejad, S., Combescure, C., Graafmans, W., Attar, H., Donaldson, L., and Pittet, D. (2011). Burden of endemic health-care-associated infection in developing countries: a systematic review and meta-analysis. Lancet. 377(9761):228-41.

Amenu, D., Belachew, T., and Araya, F. (2011). Surgical site infection rate and risk factors among obstetric cases of Jimma University Specialized Hospital, Southwest Ethiopia. Ethiopian Journal of Health Sciences. 21(2):91-100.

Bjorklund, K., Mutyaba, T., Nabunya, E., and Mirembe, F. (2005). Incidence of postcesarean infections in relation to HIV status in a setting with limited resources. Acta Obstetricia et Gynecologica Scandinavica. 84:967-971.

Caldwell, A., Young, A., Gomez-Marquez, J., and Olson, K.R. (2011). Global health technology 2.0. IEEE Pulse. 11:63-67.

Crolla, R.M., van der Laan, L., Veen, E.J., Hendriks, Y., van Schendel, C., and Kluytmans, J. (2012). PLoS One. 7(9):e44599.

Edmiston, C.E., Seabrook, G.R., Cambria, R.A., et al. (2005). Molecular epidemiology of microbial contamination in the operating room environment: is there a risk for infection. Surgery. 138(4):573-582

Eriksen, H.M., Chugulu, S., Kondo, S., and Lingaas, E. (2003). Surgical-site infections at Kilimanjaro Christian Medical Center. J Hosp Infect. 55(1):14-20.

Nejad, S.B., Allegranzi, B., Syed, S.B., Ellis, B., and Pittet, D. (2011) Health-care-associated infection in Africa: a systematic review. Bulletin of the World Health Org. 89:757-765. 
Ortega, G., Rhee, D.S., Papandria, D.J., Yang, J., Ibrahim, A.M., Shore, A.D., Makary, M.A., and Abdullah, F. (2011). An evaluation of surgical site infections by wound classification system using the ACS-NSQIP. J Surg Res. 174(1):33-38.

Sanou, J., Traore, S.S., Lankoande, J., Quedraogo, R.M., and Sanou, A. (1999). Survey of nosocomial infection prevalence in the surgery department of the Central National Hospital of Ouagadougou. Dakar Med (abstract only). 44(1):105-108.

Stevenson, K. and Cather, C. (2008). Pursuing cleanliness in a field surgical environment. AORN Journal. 87(2):306-309.

Taye, M. (2005). Wound infection in Tikur Anbessa hospital, surgical department. (2005). Ethiop Med J (abstract). 43(3):167-174.

van der Slegt, J., van der Laan, L., Veen, E.J., Hendriks, Y., Romme, J., Kluytmans, J. (2013). PLoS One. 8(8):e71566.

Whyte, W., Hodgson, R., and Tinkler, J. (1982). The importance of airborne bacterial contamination of wounds.

Journal of Hospital Infection. 3:123-135.

\section{Online document}

(2008). Promoting essential surgery in low-income countries: a hidden, cost-effective treasure.

Disease Control Priorities Project. http://www.dcp2.org/file/158/dcpp-surgery.pdf. Accessed 25

February 2012.

(2015). Global Surgery 2030: Evidence and solutions for achieving health, welfare, and economic development. The Lancet Commission on Global Surgery.

http://www.globalsurgery.info/wp-content/uploads/2015/01/Overview_GS2030.pdf. Accessed 12 May 2015.

Klingler, G.A. (1972). Digital computer analysis of particle size distribution in dusts and powders. Resource document. National Technical Information Service. http://www.dtic.mil/cgibin/GetTRDoc?Location $=\mathrm{U} 2 \& d$ doc $=$ GetTRDoc.pdf\&AD $=$ AD0752209. Accessed 20 October 2015. 